\title{
BMJ Open Staying Quit After Release (SQuARe) trial protocol: a randomised controlled trial of a multicomponent intervention to maintain smoking abstinence after release from smoke-free prisons in Victoria, Australia
}

To cite: Young JT, Puljević C, Love AD, et al. Staying Quit After Release (SQuARe) trial protocol: a randomised controlled trial of a multicomponent intervention to maintain smoking abstinence after release from smoke-free prisons in Victoria, Australia. BMJ Open 2019;9:e027307. doi:10.1136/ bmjopen-2018-027307

- Prepublication history for this paper is available online. To view these files, please visit the journal online (http://dx.doi. org/10.1136/bmjopen-2018027037).

Received 16 0ctober 2018 Revised 25 March 2019 Accepted 24 April 2019

Check for updates

(C) Author(s) (or their employer(s)) 2019. Re-use permitted under CC BY-NC. No commercial re-use. See rights and permissions. Published by BMJ.

For numbered affiliations see end of article.

Correspondence to Jesse T Young;

jesse.young@unimelb.edu.au

\section{ABSTRACT}

Introduction Smoke-free policies have been introduced in prisons internationally. However, high rates of relapse to smoking after release from prison indicate that these policies typically result in short-term smoking cessation only. These high rates of relapse, combined with a lack of investment in relapse prevention, highlight a missed opportunity to improve the health of a population who smoke tobacco at two to six times the rate of the general population. This paper describes the rationale and design of a randomised controlled trial, testing the effectiveness of a caseworker-delivered intervention promoting smoking cessation among former smokers released from smokefree prisons in Victoria, Australia.

Methods and analysis The multicomponent, brief intervention consists of behavioural counselling, provision of nicotine spray and referral to Quitline and primary care to promote use of government-subsidised smoking cessation pharmacotherapy. The intervention is embedded in routine service delivery and is administered at three time points: one prerelease and two postrelease from prison. Control group participants will receive usual care. Smoking abstinence will be assessed at 1 and 3 months postrelease, and confirmed with carbon monoxide breath testing. Linkage of participant records to survey and routinely collected administrative data will provide further information on postrelease use of health services and prescribed medication.

Ethics and dissemination Ethical approval has been obtained from the Corrections Victoria Research Committee, the Victorian Department of Justice Human Research Ethics Committee, the Department of Human Services External Request Evaluation Committee and the University of Melbourne Human Research Ethics Committee. Results will be submitted to major international health-focused journals. In case of success, findings will assist policymakers to implement urgently needed interventions promoting the maintenance of prisoninitiated smoking abstinence after release, to reduce the health disparities experienced by this marginalised population.

\section{Strengths and limitations of this study}

- The multicomponent intervention is guided by evidence supporting the effectiveness of combined behavioural counselling and pharmacotherapy for achieving smoking cessation.

- As the intervention has been embedded in routine practice with an existing, statewide service provider, it would be scalable and easily implemented as standard procedure.

- As intervention and control group participants cannot be separated, the possibility of contamination cannot be excluded.

- Although some participants may be lost to follow-up, this will be reduced by caseworkers retaining participants as clients, and prospective data linkage will provide additional follow-up data.

Trial registration number ACTRN12618000072213; Preresults.

\section{INTRODUCTION}

Tobacco smoking is the leading cause of preventable death worldwide, ${ }^{1}$ responsible for more than 7 million deaths per year. ${ }^{2}$ Despite a decrease in tobacco use in the general population in most countries, ${ }^{3}$ some population groups, including people who experience incarceration, continue to smoke at high levels. ${ }^{4-6}$ Smoking rates are extremely high among people entering prison, with point prevalence estimates ranging from $56 \%$ in the $\mathrm{USA}^{7}$ to $74 \%$ in Australia ${ }^{6}$; a rate approximately six times higher than among the general Australian population. ${ }^{8}$ Despite high rates of tobacco use, almost half of 
people entering prison in Australia who smoke express a desire to quit. $^{9}$

People in prison experience disproportionate rates of smoking-related health problems compared with their community counterparts, including cancer, chronic obstructive pulmonary disease and cardiovascular disease. ${ }^{10-12}$ These smoking-related health disparities are often compounded by entrenched social and financial disadvantage. ${ }^{7}$ Key groups within the prison population may be particularly at risk. For example, Indigenous Australians are over-represented in prisons, ${ }^{9}$ and Indigenous people in prison experience increased smoking-related health inequalities compared with both non-Indigenous people in prison and Indigenous people in the community. ${ }^{9} 1314$ Targeting and tailoring prevention strategies and interventions to priority groups has been recommended to reduce smoking-related health burden at the population level. ${ }^{15}$

In an effort to reduce exposure of staff, people who are incarcerated, and visitors to environmental tobacco smoke, which is as harmful as smoking itself, ${ }^{16}{ }^{17}$ correctional authorities have been implementing smoke-free policies that prohibit tobacco smoking on prison grounds for both people who are incarcerated and prison staff. ${ }^{4} 18$ Such policies have been introduced in prisons in New Zealand, ${ }^{19}$ several European countries, ${ }^{5}{ }^{20}$ Canada, ${ }^{21}$ most states in the USA ${ }^{4}$ and are being introduced across prisons in England and Wales. ${ }^{22}$ In Australia, smoke-free policies have been implemented in prisons in all states and territories except Western Australia, South Australia and the Australian Capital Territory. ${ }^{18}$

Despite good evidence that smoke-free policies result in health benefits for people in prison, ${ }^{23} 24$ including significant reductions in smoking-related deaths, ${ }^{7}$ they do not ensure complete abstinence for all people in prisons. The prevalence of any level of tobacco use in smoke-free prisons has been reported between $20 \%$ and $76 \%,{ }^{4}$ however no study has reported this prevalence in an Australian prison. Research conducted in the USA has found that between $60 \%{ }^{25}{ }^{26}$ and $74 \%^{27}$ of former smokers released from smoke-free prisons relapse to smoking within 24 hours of release, and $97 \%$ relapse within 6 months of release. ${ }^{26}$ Similarly, in a recent Australian study, $72 \%$ of people released from smoke-free prisons relapsed to smoking on the day of release, and $94 \%$ relapsed within 2 months of release. ${ }^{28}$ A systematic review $^{29}$ of prison-based smoking cessation interventions found that correctional smoke-free policies result in short-term smoking abstinence only and, on their own, are insufficient to maintain long-term smoking cessation after release from prison.

The high rates of relapse to smoking among people leaving prison are especially concerning given that people released from prison experience particularly poor physical $^{30} 31$ and mental ${ }^{32} 33$ health outcomes compared with their community counterparts, including significantly higher rates of smoking-related illness. ${ }^{12} 30$ Prison sentences typically exceed the period required for nicotine withdrawal, ${ }^{34}$ such that prison-initiated abstinence lays the groundwork for providing postrelease relapse prevention support, and improving smoking and related health outcomes for this highly marginalised group. However, due to the significant challenges faced by many people returning from prison to the community, including securing housing and employment, ${ }^{35} 36$ relapse to substance dependence ${ }^{37} 38$ and re-establishing interpersonal relationships, ${ }^{39}$ maintaining abstinence from smoking may not be a high priority. ${ }^{40}$

Despite the proliferation of smoke-free policies in prisons globally, there is a lack of high-quality research in this area. A recent systematic review ${ }^{41}$ identified only 15 studies reporting on smoking intentions or behaviours following release from smoke-free prisons, almost exclusively conducted in the USA. Additionally, we are aware of only one randomised controlled trial (RCT) ${ }^{25}{ }^{41}-$ the US-based Working Inside for Smoking Elimination (WISE) project ${ }^{25}$ - which evaluated the effectiveness of a prerelease intervention $(6 \times 1$ hour sessions of individual behavioural counselling) aimed at promoting smoking abstinence following release from a smoke-free prison. At 3 months postrelease, $12 \%$ of the intervention group remained abstinent from smoking compared with only $2 \%$ of the control group, showing a modest yet significant effect. ${ }^{25}$ Evidence-based interventions aimed at preventing relapse to smoking after release from smokefree prisons, such as the WISE study ${ }^{25}$ are urgently needed to reduce the substantial health inequalities experienced by this marginalised group, and to ensure that the potential public health benefits of prison smoke-free policies are realised. An ideal model would provide continuity of care from prerelease to postrelease support. To date, no RCT has investigated the effect of a prerelease and postrelease intervention aimed at maintaining abstinence from smoking in people released from prison. This study will assess the impact of a smoking cessation intervention that is initiated prerelease and delivered during the high-risk postrelease period.

In preparation for this project, we collaborated with the Australian Community Support Agency (ACSO) in 2016 and conducted a 5-month pilot study with people due for release from smoke-free prisons in Victoria, Australia. ${ }^{42}$ Among the 54 participants recruited by caseworkers, $85 \%$ were smokers before prison entry, averaging 19 cigarettes per day. Caseworkers found it acceptable and feasible to deliver a brief smoking cessation intervention (3-5 min conversation based on principles of motivational interviewing $^{43}$ ) to clients prerelease and/or at the first postrelease appointment. After receiving the brief intervention, the majority of participants $(67 \%)$ reported an intention to change their smoking behaviour; consisting of $43 \%$ who were interested in remaining abstinent postrelease, and $24 \%$ who wanted to reduce the amount they smoked prior to prison. Among participants who were contacted at 1 month postrelease, almost all had returned to smoking. These pilot findings suggested that more intensive support is required to reduce postrelease 
relapse to smoking and to encourage those who relapse to make further quit attempts, consistent with research demonstrating a dose-dependent relationship between increased attendance at cessation sessions and smoking abstinence in marginalised groups. ${ }^{44}$

Using a randomised design in a cohort of previously smoking adults recently released from smoke-free prisons in Victoria, Australia, this study aims to evaluate the effects of an intervention involving (1) a brief caseworker-facilitated behavioural intervention encouraging postrelease smoking abstinence, (2) provision of nicotine replacement therapy (NRT) mouth spray to manage cravings and (3) and referral to abstinence supports (Quitline, and general practitioners (GPs) for prescription of smoking cessation pharmacotherapy (SCP)) following release from smoke-free prisons, on smoking abstinence.

\section{METHODS AND ANALYSIS \\ Trial design}

The Staying Quit After Release (SQuARe) trial is a two-arm, double-blinded, multisite RCT evaluating the impact of a multicomponent smoking cessation support intervention promoting maintained smoking abstinence following release from smoke-free prisons. The primary outcomes are i) continuous abstinence (prevention of smoking relapse) and ii) 7-day point prevalence abstinence (recovery from relapse), assessed at 1 and 3 months after release from prison using follow-up surveys and confirmed with carbon monoxide $(\mathrm{CO})$ breath testing for those who consent. Secondary outcomes include time to smoking relapse, intention to quit, quit attempts and tobacco consumption as well as the number of brief interventions received and utilisation of recommended abstinence supports (number of Quitline calls, GP appointments and use of SCP within 3 months of release). Participant records will also be prospectively linked with routinely collected administrative data. We used the Standard Protocol Items: Recommendations for Interventional Trials checklist when drafting this protocol. ${ }^{45}$ The design of the trial is depicted in figure 1.

\section{Study setting}

This study will operate in partnership with ACSO's ReConnect programme across seven prisons in Victoria: Barwon Prison, Dame Phyllis Frost Centre, Fulham Correctional Centre, Karreenga Prison, Loddon Prison Precinct, Marngoneet Correctional Centre and Port Phillip Prison. These prisons represent both male-only and female-only prisons, receive people from urban and regional areas, and include all security ratings. The ACSO ReConnect programme provides reintegration outreach services to people being released from prisons in Victoria. ReConnect support services are initiated up to 6 weeks prior to release, continuing up to 12 months postrelease, and are specifically targeted to people who have been convicted of serious violent or sex offences, Aboriginal and Torres Strait Islander people, women and people with complex transitional needs (such as those with intellectual disability, mental illness, homelessness and/or a history of substance dependence). Those deemed eligible for the ReConnect programme are assigned an ACSO caseworker who assists them to develop goals and a transition plan which includes linking them to additional support services tailored to their needs. All prisons in the state of Victoria have been smoke-free since 1 July 2015.

\section{Eligibility criteria}

Embedded in normal service delivery, ACSO caseworkers will screen clients in the seven participating prisons for eligibility and interest in participating in the research by asking the following questions: (1) "Before you came into prison were you smoking tobacco (at least one cigarette a week or at least 100 cigarettes or the equivalent amount of tobacco in their lifetime)?"; (2) "Do you think that you might be required to wear a Secure Continuous Remote Alcohol Monitoring (SCRAM) bracelet after release from prison?"; (3) "Are you interested in meeting with researchers to learn more and potentially participate in the study?" Those eligible for the trial will be: (1) clients of ACSO's ReConnect programme; (2) adults (aged $\geq 18$ years); (3) within 4-6 weeks of expected release from prison; (4) regular tobacco smokers $(\geq 1$ cigarette/week and $\geq 100$ cigarettes or the equivalent amount of tobacco in their lifetime) prior to prison entry and (5) able to provide informed, written consent to participate. Individuals who are expected to be issued with a SCRAM bracelet on release will be ineligible, as NRT spray contains a trace amount of ethanol, which may be detected by the bracelet.

\section{Recruitment and participant timeline}

Using a secure project email address, ACSO caseworkers will alert the research coordinator (the only unblinded member of the research team) of potentially eligible clients. The research coordinator will then arrange for a research assistant (RA) to approach the client in prison, confirm their eligibility, explain the study and obtain written, informed consent. To reduce response burden for participants, consent will also be sought to access their health, substance use and psychosocial information from ReConnect case files in ACSO's administrative database. All participants will be asked to provide consent for the release of Quitline data (number, length and timing of calls) and for person-level Medicare Benefits Schedule (MBS; primary care billing records) and Pharmaceutical Benefits Scheme (PBS; medication dispensing records) data from the Commonwealth Department of Human Services (DHS), with the purpose of collecting data regarding primary care contact and medication dispensing for up to 2 years following release from prison.

Individuals who consent to participate will complete a baseline survey. To minimise literacy concerns, the survey will be verbally administered by a trained RA and is expected to take approximately $20 \mathrm{~min}$ to complete. In order to permit assessment of selection bias and 


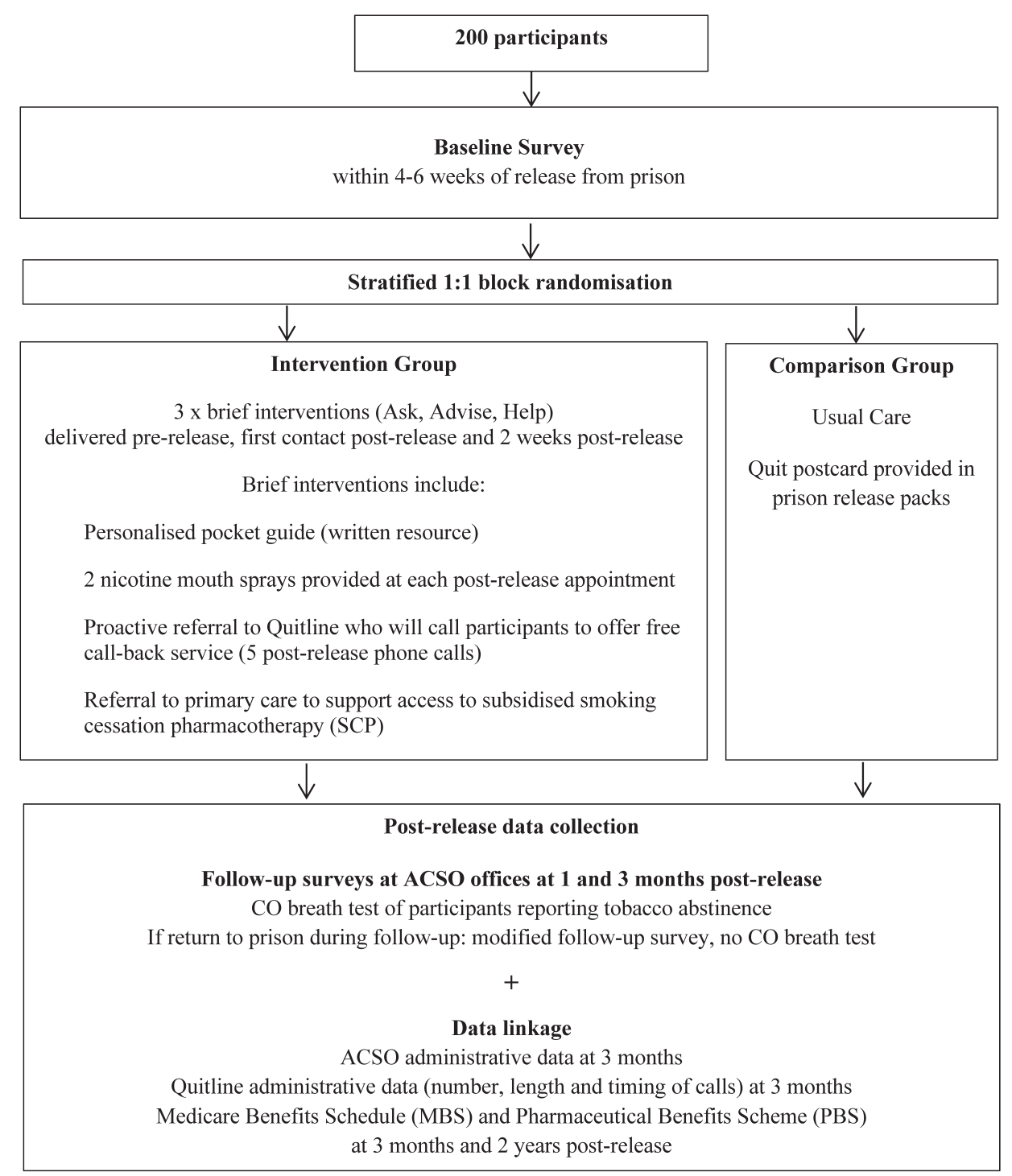

Figure 1 Overview of study design. ACSO, Australian Community Support Organisation; CO, carbon monoxide.

calculation of a recruitment fraction, ACSO will provide the research team with routinely collected de-identified, aggregate demographic information for everyone screened by ACSO staff for participation.

Follow-up interviews will be conducted by a trained RA, blinded to experimental condition, with all participants at 1 and 3 months after release from prison. Face-to-face follow-up interviews will take place at ACSO offices or other appropriate community locations. In cases where this is not feasible (eg, due to distance or safety concerns), interviews will be completed by telephone. Participants will be reimbursed $\$ A 20$ for each completed interview (sent via post as a secure money order for those interviewed by phone). If a participant is re-incarcerated during follow-up, interviews will be conducted in prison, but the participant will not receive reimbursement, as per Corrections Victoria policy.

\section{Randomisation}

At conclusion of the baseline assessment, the RA will send an email to the (unblinded) research coordinator using the secure project email address, listing the participant's date of baseline interview, date of expected release from prison, intention to remain abstinent after release (yes/no) and Corrections Reference Number (CRN, a unique number assigned to all people entering the correctional system). Given evidence that people who are incarcerated who intend to remain quit are more likely to remain abstinent postrelease, ${ }^{40}$ randomisation will be stratified by intention to remain abstinent, using random permuted blocks of sizes four and six. An equal number of participants will be assigned to the intervention and usual care groups. Randomisation will be independently managed by the research coordinator, such that the RAs who interview participants will remain blinded to intervention group status. The intervention group identifier will be assigned at random and will not be identified during data analysis, such that data analysts will also remain blinded. 


\section{Intervention}

Intervention development was guided by evidence ${ }^{46} 47$ that cessation rates are maximised when brief intervention from a health or support worker links clients to both a multisession specialist behavioural intervention (such as Quitline) and pharmacotherapy (nicotine products or cessation medication). Participants randomised to the intervention group will receive a multicomponent intervention consisting of: (1) a caseworker-facilitated brief intervention encouraging postrelease smoking abstinence, (2) provision of NRT spray to manage cravings and (3) proactive referral to abstinence supports (Quitline, and GPs for prescription of SCP) following release from smoke-free prisons.

\section{Brief intervention}

Prior to commencement of participant recruitment, ACSO caseworkers will receive interactive training on how to deliver a brief, evidence-based, multicomponent intervention sessions, designed by Quit Victoria (authors DB and CS), to support clients to remain smoke-free after release from prison. Following randomisation, ACSO staff will provide intervention group participants with a brief $(<10 \mathrm{~min})$ intervention at three time points: 1) between the prerelease baseline assessment and release from prison; 2) on the first day after release from prison, or as soon as practicable thereafter and 3) 2 weeks after release from prison. The aims of the brief intervention are to raise the issue of smoking, encourage openness to the possibility of staying smoke-free and to link participants to effective forms of cessation help. Each brief intervention will follow a three-step format: Ask (about current smoking behaviour and intentions; Advise (provide personalised advice to promote smoking abstinence postrelease, including promoting the benefits of cessation, normalisation of postrelease increase in cravings and encouraging use of behavioural strategies, Quitline or nicotine spray) and Help (provision of nicotine spray, personalised pocket guide and referrals to Quitline and primary care). ACSO caseworkers will inform the trial coordinator on the completion (or not) of each of the three brief interventions, to permit exploration of intervention fidelity.

During the first intervention contact (before release), participants will be provided with a personalised, interactive self-help pocket guide (figure 2) that encourages smoking cessation postrelease and lists a 1800 (free call) Quitline telephone number specifically created for this study. ACSO caseworkers will assist participants in filling out the pocket guide during the first prerelease intervention contact. To minimise contamination, the ACSO worker will keep the personalised pocket guide until the second intervention contact, on the day of release, where it will be provided to the participant. On the day of release, the ACSO caseworker will review the pocket guide content with the participant, and will encourage the participant to continue reviewing the pocket guide over subsequent weeks. Furthermore, prior to study recruitment, ACSO caseworkers will undergo training in research methodology, delivered by the research team, which will clearly define usual care and address the potential for contamination from providing the intervention to the comparison group.

\section{Quitline telephone cessation service}

During the second (day of release) intervention, caseworkers will send a referral form to Quitline who will call participants to offer their free counselling call-back service. Intervention group participants will receive these calls on five suitable occasions during the 28 days after release from prison. Evidence suggests that call-back telephone counselling significantly increases the likelihood of quitting smoking. ${ }^{49}$ Quitline is experienced with assisting people in prison and other vulnerable groups with complex needs, ${ }^{50}$ and all Quitline staff are trained in World Health Organization telephone counselling guidelines. ${ }^{51}$ Quitline also offers a culturally sensitive Indigenous Quitline staffed by Indigenous Quit Specialists. Quitline counselling typically focuses on building participants' motivation and confidence to (stay) quit via provision of psychoeducation and skills building, including identifying triggers to smoke, exploring alternative strategies to remain abstinent and helping people to optimise their use of NRT products or SCP.

\section{NRT mouth spray}

Intervention group participants will receive two NRT mouth sprays (each containing 150 sprays $\times 1 \mathrm{mg}$ nicotine) at time 2 (day of release) and again at time 3 (2 weeks postrelease), provided by ACSO staff. NRT offers a concrete alternative to smoking with proven effectiveness and few adverse side effects, ${ }^{52}$ and is likely to assist participants with managing the increase in tobacco cravings that they are likely to experience on release from prison, driven by the availability of tobacco and encountering situations and emotions that are strongly associated with smoking. ${ }^{41}$ Nicotine mouth spray has advantages over other forms of NRT, as it is easy to use and provides faster craving relief. ${ }^{5354}$ However, short-acting forms of nicotine replacement (such as NRT spray) are not subsidised by the Australian $\mathrm{PBS}^{55}$ (a programme that allows Australian citizens and permanent residents to access government-subsidised prescription medications) and the cost of NRT is often cited by smokers from disadvantaged groups as a barrier to use. ${ }^{56}$ Participants will also be provided with a credit card-sized sheet of laminated paper with information and instructions for correct and safe use of the NRT spray.

\section{Referral to primary care}

At the first intervention contact (prior to release from prison), ACSO caseworkers will identify a suitable government-subsidised (known as bulk-billing in Australia) primary care practice for each participant, and will encourage or assist the participant to make an appointment with a GP to discuss further options for accessing government-subsidised smoking cessation pharmacotherapy. At each postrelease brief intervention, the ACSO 


\section{Staying off the smokes: Nowit's my choice.}

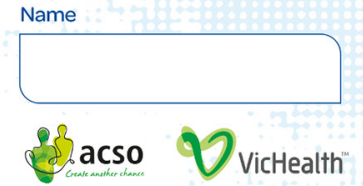

73 $\frac{\text { THE UnIVERSITY OF }}{\text { MELBOURNE }}$

\section{Quitline} 1800960788

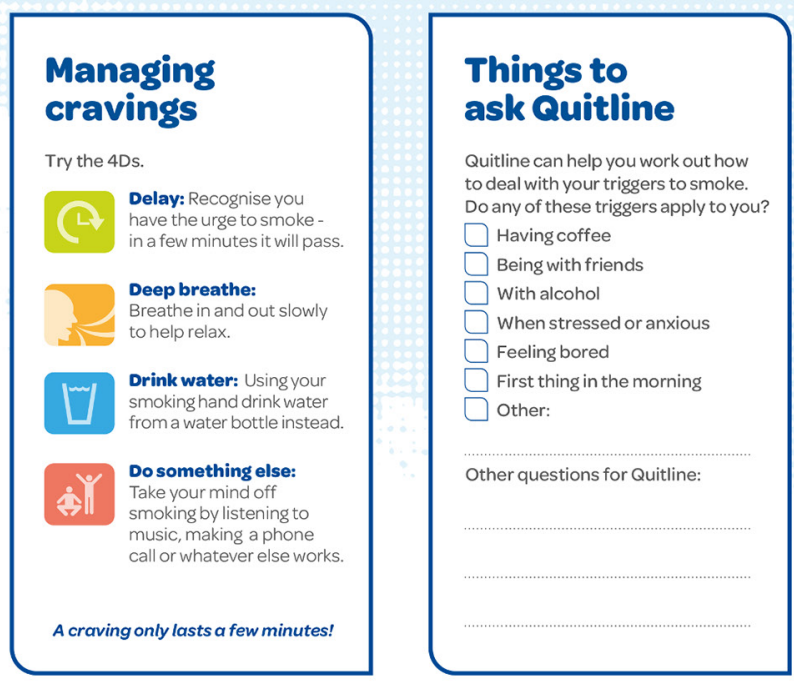

Figure 2 Personalised, interactive self-help pocket guide.

caseworker will ask the participant if they have accessed primary care since release from prison, and if not, they will provide a further referral to the GP.

In Australia, nicotine patches (ATC code N07BA01) and the stop-smoking medications varenicline (ATC code N07BA03) and bupropion (ATC code N06AX12) are available via prescription at discounted rates through the PBS. ${ }^{57}$ For participants who return to smoking, combination therapy (nicotine patches combined with a short-acting form of NRT such as nicotine spray) is a recommended first-line option for nicotine-dependent smokers. ${ }^{58}$ For example, concessional users, including most people released from prison, can access 4 weeks' supply of nicotine patches for $\$ A 6.40$ vs a minimum of $\$ A 60$ without PBS subsidy. ${ }^{59}$ Despite the low cost of smoking cessation pharmacotherapy through this scheme, previous research has found that fewer than 1 in 10 prior smokers accesses PBS-subsidised pharmacotherapy following release from prison. ${ }^{60}$ Furthermore, passive referrals to primary care for people released from prison increase contact with primary care ${ }^{61}$ and this contact has collateral benefits for

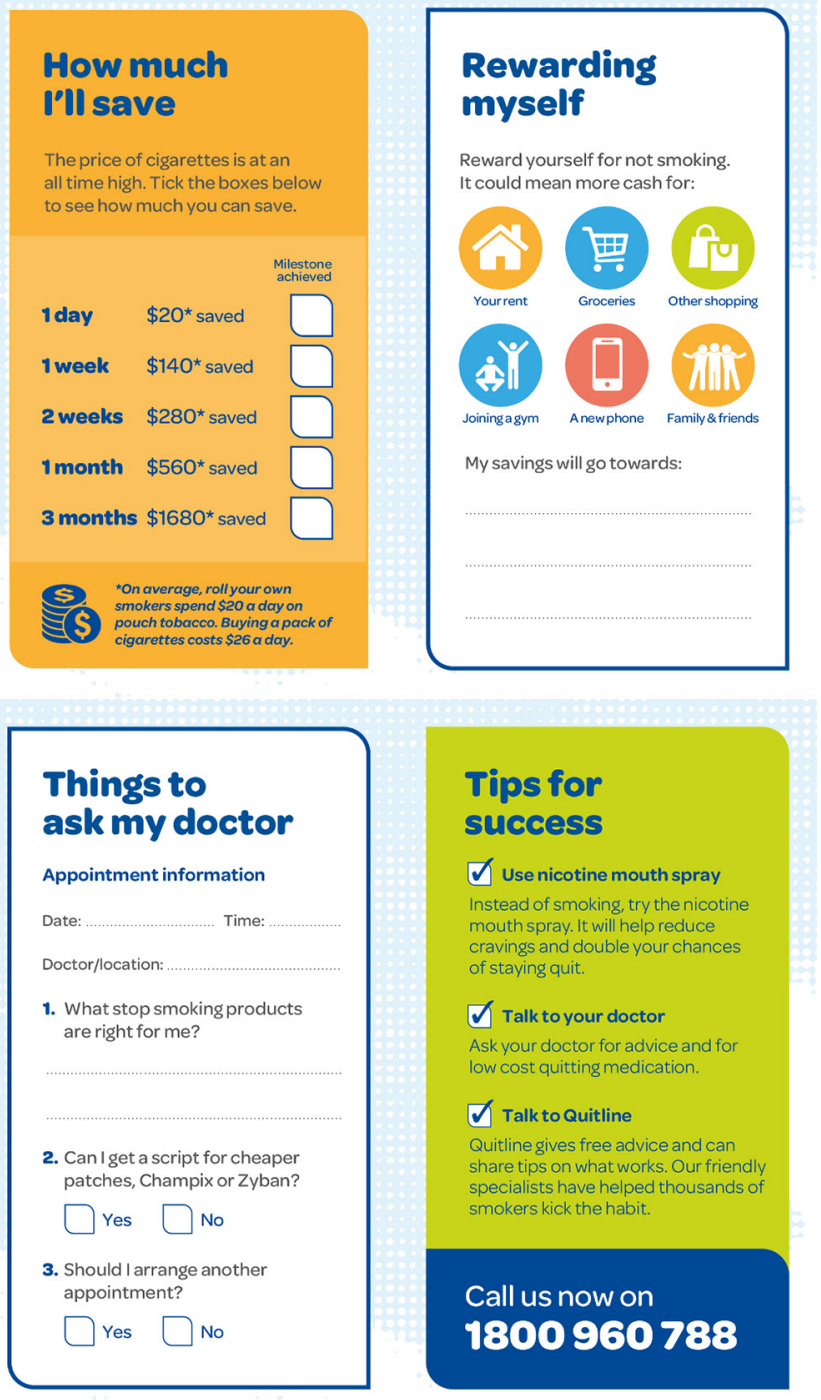

engagement with mental health and alcohol and other drug treatment services ${ }^{62}$; services that can also be subsidised through the MBS.

\section{Comparison group}

Participants assigned to the comparison group will receive usual care from ACSO caseworkers which includes providing all clients (in their release pack) with a Quit Victoria postcard that illustrates what could be bought with the money saved by not smoking, and encourages calls to the Quitline phone number. If requested by participants, ACSO caseworkers will provide brief advice and recommend that the participant calls Quitline, although we expect that few will do so as smokers are often reluctant to seek cessation support. ${ }^{6364}$

\section{Survey measures}

The baseline survey will assess smoking behaviours, quit attempts and use of smoking cessation pharmacotherapy and behavioural support before prison entry, and intention, confidence and social support to remain abstinent 
following release from prison. All baseline survey questions are either validated scales or designed by the authors, based on previous research. ${ }^{25}{ }^{28}$ Risky alcohol use before entry to prison will be assessed using the AUDIT-C. ${ }^{65}$ Illicit drug use (cannabis, methamphetamines, heroin and other opioids) in the 3 months before entry to prison will be assessed using the ASSIST. ${ }^{66}$ Current smoking-related moods and experiences will be measured using the Minnesota Nicotine Withdrawal Scale. ${ }^{67}$ Current psychological distress will be assessed using the K10. ${ }^{68}$ Self-report questions designed by the research team will assess current general physical health. From a 6-point scale, intention to remain abstinent will be coded yes if the participant selects "I want to stop smoking but didn't think about it until coming to prison"; "I really want to stop smoking and hope to stay smoke free after release" or "I really want to stop smoking and plan to stay smoke free after release". Intention to remain abstinent will be coded no if the participant selects "I don't know"; "I don't want to stop smoking" or "I think I should stop smoking but don't really want to".

The follow-up survey, administered at both 1 and 3 months following release from prison, will include the same measures as the baseline survey and will assess current smoking status and smoking behaviour; number of days before resuming tobacco smoking following release and length of any subsequent quit attempts (using the timeline follow-back method ${ }^{69} 70$ ); intention, motivation and self-efficacy to stop smoking/remain abstinent and use and perceived utility of SCP and behavioural support. Participants who return to prison during the follow-up period will undertake a slightly modified follow-up survey in prison. Participants interviewed in prison or by telephone will not undergo a CO breath test. As some research has suggested that self-report may be an unreliable measure of smoking abstinence, ${ }^{71}$ we will use $\mathrm{CO}$ test results to assess the reliability of self-reported abstinence, thereby informing our interpretation of self-reported abstinence among participants interviewed over the phone.

\section{Administrative data linkage}

Baseline survey data will be deterministically linked to ACSO administrative data, using each participant's CRN as a unique identifier, and then probabilistically linked to person-level MBS and PBS records. Relevant measures from the ACSO administrative data include dates of client contact with caseworker prerelease and postrelease; demographic information; current legal status; arrests, emergency department presentations, hospitalisations, overdoses and injecting drug use in the 4 weeks prior to ACSO contact; lifetime history of traumatic brain injury and chronic physical health conditions; current mental health diagnoses and a suicide and self-harm risk assessment. Quitline will provide administrative data on the number, length and timing of calls provided to participants during the 3 months following their release from prison.
The MBS and PBS are national registers administrated by the DHS and include information on all Australian citizens' and permanent residents' use of primary health services subsidised through the MBS, and the dispensing of subsidised prescription medication through the PBS. Available and relevant MBS variables include date of service, item number, item description, cost to the patient, scrambled rendering provider number and rendering provider postcode. Relevant PBS variables include date of supply, date of prescription, PBS item code, item description, patient category, cost to patient, ATC code, scrambled prescriber number and form category (ie, original or repeat prescription).

Three months after the last participant is released from prison, a list of participants who consented to data linkage will be provided to DHS using each participant's CRN as a unique identifier. DHS will then use probabilistic data linkage to link participant identities with their MBS and PBS records which we will combine with survey data using the CRN.

Probabilistic data linkage is considered the gold-standard method, with validation of linkage with state health records producing a false linkage rate of $<0.1 \%{ }^{72}$ Similar methods have been used for linkage of correctional records with national mortality data in Australia, ${ }^{32}$ 73-79 with excellent sensitivity and specificity. ${ }^{81}$ To assess long-term access to primary care and subsidised smoking cessation medication, a second data linkage to MBS and PBS records will be conducted 2years following the last participant's release from prison.

\section{Outcomes}

There are two primary outcomes for the study. The first is continuous abstinence at 3 months postrelease, defined as the combination of: i) not relapsing (answering 'no' to the question, "Have you smoked cigarettes for 7 days in a row at any time since leaving prison?" ${ }^{82}$; ii) reporting 7-day point prevalence abstinence (answering 'no' to the question, "Have you smoked a cigarette at all, even a puff, in the last 7 days?") and iii) biochemical verification of point prevalence abstinence evaluated using a $\mathrm{CO}$ breath test on a piCO+ ${ }^{\mathrm{TM}}$ Smokerlyzer $\circledast$, with an expired breath $\mathrm{CO}$ level of $<10 \mathrm{ppm}$ considered indicative of abstinence. ${ }^{83}$

Given that many people being released from smokefree prisons may smoke one or a few cigarettes on the day of release to celebrate freedom, ${ }^{84}$ measures of continuous abstinence may under-ascertain intervention effects, hence the second primary outcome is recovery from relapse at the 3-month follow-up, defined as the combination of i) reporting 7-day point prevalence abstinence, that is, answering 'no' to the question, "Have you smoked a cigarette at all, even a puff, in the last 7 days?" and ii) biochemical verification of point prevalence abstinence as described above.

Secondary outcomes assessed at 3 months postrelease will include smoking-related measures (time to smoking relapse, intention to quit, quit attempts and tobacco consumption), and smoking cessation support received 
(including number of brief interventions received for intervention group participants, and utilisation of recommended abstinence supports including number of Quitline calls, primary care appointments and use of smoking cessation pharmacotherapy), ascertained from both self-report and routinely collected administrative data.

\section{Sample size}

This is a pilot study and as such sample size is determined by feasibility considerations, rather than power calculations. Based on our earlier pilot work and throughput data provide by ACSO, we anticipate recruiting 200 participants over a 12-month period. This sample size is sufficient to assess the feasibility and acceptability of the procedures and measures, and to provide preliminary evidence of the impact of the intervention on smoking relapse following release from prison. Nevertheless, this sample size also provides $80 \%$ power to detect an increase in abstinence of $10 \%$ (2\% control vs $12 \%$ intervention with a one-tailed test with a $95 \% \mathrm{CI}, \alpha=0.05$ ).

\section{Statistical analysis}

The primary analysis will be an intention-to-treat analysis (ie, by group assignment regardless of the actual level of smoking cessation support received) conducted on those with follow-up data. Based on previous research with this population, we anticipate a follow-up fraction of around $80 \% .{ }^{61}{ }^{85}$ In a priori sensitivity analyses, we will (a) treat those lost to follow-up as relapsed, and (b) use multiple imputation to impute missing outcome values. ${ }^{86}$ We will also undertake an a priori per-protocol analysis in which we will exclude individuals in the intervention group who do not receive the intervention (defined as receiving at least one brief intervention within 28 days of release from prison). Potential selection bias will be assessed by comparing the demographic characteristics of our study group with the aggregate characteristics of all ACSO ReConnect clients during the recruitment period.

We will calculate descriptive statistics for all measures. We will construct generalised linear mixed models (logistic regression for dichotomous outcomes, and linear regression for continuous outcomes) to test whether differences between treatment groups persist after adjusting for correlation between repeated measures and for other predictors of quit success identified from relevant literature. We will report the treatment effect as the absolute difference in smoking abstinence between groups at each follow-up time point. In addition, we will explore a possible dose-response relationship between the amount of cessation support received (behavioural and/or pharmacological) and abstinence.

\section{PATIENT AND PUBLIC INVOLVEMENT}

Participants and the public were not involved in the development of the research question, design or conduct of this study, and did not assess the potential burden of the intervention in this study. However, we will disseminate a summary of study findings to participants who requested this information on enrolment to the study.

\section{ETHICS AND DISSEMINATION}

This study has received approval from the Corrections Victoria Research Committee, the Victorian Department of Justice Human Research Ethics Committee (ref: CF/17/27939), the Department of Human Services External Request Evaluation Committee (ref: MI8417) and the University of Melbourne Human Research Ethics Committee (ref: 1851417). The trial is registered with the Australian New Zealand Clinical Trials Registry (ACTRN12618000072213). All electronic data will be entered into a secure, password-protected electronic database on a server at the University of Melbourne. Hard copies of participant consent forms, surveys and photocopies of pocket guides completed by participants in the intervention group will be kept in secure, locked filing cabinets at the University of Melbourne. Consent forms, surveys and photocopies of pocket guides will all be kept separately to avoid potential participant identification.

After analysis, a summary of findings will be sent to Corrections Victoria and VicHealth, and to participants who requested this information. The research team will submit findings to major international peer-reviewed health and substance use-focused journals. Any protocol modifications will be published on the ANZ Clinical Trials initial registration ACTRN12618000072213.

\section{Author affiliations}

${ }^{1}$ Melbourne School of Population and Global Health, The University of Melbourne, Melbourne, Victoria, Australia

${ }^{2}$ Centre for Adolescent Health, Murdoch Children's Research Institute, The University of Melbourne, Melbourne, Victoria, Australia

${ }^{3}$ School of Population and Global Health, The University of Western Australia, Perth, Western Australia, Australia

${ }^{4}$ National Drug Research Institute, Curtin University, Perth, Western Australia, Australia

${ }^{5}$ Centre for Health Services Research, University of Queensland, Brisbane, Queensland, Australia

${ }^{6}$ Griffith Criminology Institute, Griffith University, Brisbane, Queensland, Australia ${ }^{7}$ Cancer Council Victoria, Melbourne, Victoria, Australia

${ }^{8}$ School of Medicine and Public Health, The University of Newcastle, Newcastle, New South Wales, Australia

${ }^{9}$ Australian Community Support Organisation, Richmond, Victoria, Australia

${ }^{10}$ School of Public Health and Preventive Medicine, Monash University, Melbourne,

Victoria, Australia

${ }^{11}$ Mater Research Institute-UQ, The University of Queensland, Brisbane, Queensland, Australia

${ }^{12}$ Netherlands Institute for the Study of Crime and Law Enforcement, Amsterdam, The Netherlands

Acknowledgements The authors would like to thank ReConnect workers from ACSO for their collaboration, and Nathan Jilich and Sian Lloyd from Quit Victoria and Ron Borland from Cancer Council Victoria for their contributions to the development of this project.

Contributors SAK, JTY, CP, ADL, EKJ, CJS, DB, RW, SP and EB contributed to the conceptualisation and design of the project. JTY, CP, ADL, EKJ, CJS, DB, RW, SP and SAK contributed to drafting and editing the manuscript. All authors approved the final manuscript.

Funding This trial is funded by a VicHealth Innovation Research Grant. 
Disclaimer The funder had no role in the study design; collection, management, analysis or interpretation of data; writing of the manuscript or the decision to submit the manuscript for publication.

Competing interests None declared.

Patient consent for publication Not required.

Ethics approval This study has received approval from the Corrections Victoria Research Committee, the Victorian Department of Justice Human Research Ethics Committee (ref: $\mathrm{CF} / 17 / 27939)$, the Department of Human Services External Request Evaluation Committee (ref: Ml8417) and the University of Melbourne Human Research Ethics Committee (ref: 1851417)

Provenance and peer review Not commissioned; externally peer reviewed.

Open access This is an open access article distributed in accordance with the Creative Commons Attribution Non Commercial (CC BY-NC 4.0) license, which permits others to distribute, remix, adapt, build upon this work non-commercially, and license their derivative works on different terms, provided the original work is properly cited, appropriate credit is given, any changes made indicated, and the use is non-commercial. See: http://creativecommons.org/licenses/by-nc/4.0/.

\section{REFERENCES}

1. Samet JM. Tobacco smoking: the leading cause of preventable disease worldwide. Thorac Surg Clin 2013;23:103-12.

2. World Health Organization. Tobacco Fact Sheet. Geneva, Switzerland 2018.

3. GBD 2015 Tobacco Collaborators. Smoking prevalence and attributable disease burden in 195 countries and territories, 19902015: a systematic analysis from the Global Burden of Disease Study 2015. Lancet 2017;389:1885-906.

4. Kennedy SM, Davis SP, Thorne SL. Smoke-free policies in U.S. Prisons and jails: A review of the literature. Nicotine Tob Res 2015;17:629-35

5. Baybutt M, Ritter C SH. Tobacco use in prison settings: a need for policy implementation. Geneva, Switzerland 2014 http://www.euro. who.int/_data/assets/pdf_file/0004/249205/Prisons-and-Health,-16 Tobacco-use-in-prison-settings-a-need-for-policy.pdf.

6. AlHW. Australia's Health. Canberra, Australia, 2016. https://www. aihw.gov.au/reports/australias-health/australias-health-2016/ contents/summary.

7. Binswanger IA, Carson EA, Krueger PM, et al. Prison tobacco control policies and deaths from smoking in United States prisons: population based retrospective analysis. BMJ 2014;349:g4542-12.

8. AlHW. National Drug Strategy Household Survey 2016 Key Findings. Canberra, Australia 2017 http://www.aihw.gov.au/alcohol-and-otherdrugs/data-sources/ndshs-2016/key-findings/.

9. AlHW. The Health of Australia's Prisoners 2015, Cat. 2015. No. PHE 207. Canberra, Australia http://www.aihw.gov.au/publication-detail/? id=60129553527.

10. Wilper AP, Woolhandler S, Boyd JW, et al. The health and health care of US prisoners: results of a nationwide survey. Am J Public Health 2009;99:666-72

11. Binswanger IA, Krueger PM, Steiner JF. Prevalence of chronic medical conditions among jail and prison inmates in the USA compared with the general population. J Epidemiol Community Health 2009;63:912-9.

12. Rosen DL, Schoenbach VJ, Wohl DA. All-cause and cause-specific mortality among men released from state prison, 1980-2005. Am J Public Health 2008;98:2278-84.

13. Richmond RL, Wilhelm KA, Indig D, et al. Cardiovascular risk among Aboriginal and non-Aboriginal smoking male prisoners: inequalities compared to the wider community. BMC Public Health 2011;11:783.

14. Indig D, Mcentyre E, Page J, et al. NSW Inmate health survey: aboriginal health report. Sydney, Australia 2009;2010 http://www. justicehealth.nsw.gov.au/publications/inmate-health-surveyaboriginal-health-report.pdf

15. Bonevski $\mathrm{B}$, Borland $\mathrm{R}$, Paul $\mathrm{CL}$, et al. No smoker left behind: it's time to tackle tobacco in Australian priority populations. Med J Aust 2017;207:141-2.

16. U.S. Department of Health and Human Services. The Health Consequences of Involuntary Exposure to Tobacco Smoke: A Report of the Surgeon General.. 2006 http://www.surgeongeneral.gov/ library/reports/secondhandsmoke/fullreport.pdf.

17. Hill S, Blakely T, Kawachi I, et al. Mortality among "never smokers" living with smokers: two cohort studies, 1981-4 and 1996-9. BMJ 2004;328:988-9.
18. Butler TG, Yap L. Smoking bans in prison: time for a breather? Med $J$ Aust 2015;203:313.

19. Bonita R, Beaglehole R. New Zealand leads the way in banning smoking in prisons. BMJ 2013;346:f3923.

20. Hartwig C, Stöver H, Weilandt C, et al. Report on Tobacco Smoking in Prison, 2008. http://ec.europa.eu/health/ph_determinants/life_ style/drug/documents/drug frep2.pdf.

21. Collier R. Prison smoking bans: clearing the air. Can Med Assoc J 2013;185:E474.

22. Woodall J, Tattersfield A. Perspectives on implementing smokefree prison policies in England and Wales. Health Promot Int 2018;33:1066-73.

23. Dickert J, Williams JM, Reeves $\mathrm{R}$, et al. Decreased mortality rates of inmates with mental illness after a tobacco-free prison policy. Psychiatr Serv 2015;66:975-9.

24. Connell RA. Tobacco-free prison policies and health outcomes among inmates. 2010 http://uknowledge.uky.edu/cgi/viewcontent. cgi?article=1021\&context=gradschool_diss.

25. Clarke JG, Stein LA, Martin RA, et al. Forced smoking abstinence: not enough for smoking cessation. JAMA Intern Med 2013;173:789-94.

26. Lincoln T, Tuthill RW, Roberts CA, et al. Resumption of smoking after release from a tobacco-free correctional facility. J Correct Health Care 2009;15:190-6.

27. Frank MR, Blumhagen R, Weitzenkamp D, et al. Tobacco use among people who have been in prison: Relapse and factors associated with trying to quit. J Smok Cessat 2016;10.

28. Puljević C, de Andrade D, Coomber R, et al. Relapse to smoking following release from smoke-free correctional facilities in Queensland, Australia. Drug Alcohol Depend 2018;187:127-33.

29. de Andrade D, Kinner SA. Systematic review of health and behavioural outcomes of smoking cessation interventions in prisons. Tob Control 2016;26:495-501.

30. Binswanger IA, Stern MF, Deyo RA, et al. Release from prison--a high risk of death for former inmates. N Engl J Med 2007;356:157-65.

31. Morrow KM. Project START Study Group. HIV, STD, and hepatitis risk behaviors of young men before and after incarceration. AIDS Care 2009;21:235-43.

32. Thomas EG, Spittal MJ, Heffernan EB, et al. Trajectories of psychological distress after prison release: implications for mental health service need in ex-prisoners. Psychol Med 2016;46:611-21.

33. Borschmann R, Thomas E, Moran P, et al. Self-harm following release from prison: A prospective data linkage study. Aust N Z J Psychiatry 2017;51.

34. Shiffman S, Patten C, Gwaltney C, et al. Natural history of nicotine withdrawal. Addiction 2006;101:1822-32.

35. Porter LC. Incarceration and Post-release Health Behavior. J Health Soc Behav 2014;55:234-49.

36. Baldry E, McDonnell D, Maplestone P, et al. Australian Prisoners' Post-release Housing. Current Issues in Criminal Justice 2003;15:155-69.

37. Winter RJ, Stoové M, Degenhardt L, et al. Incidence and predictors of non-fatal drug overdose after release from prison among people who inject drugs in Queensland, Australia. Drug Alcohol Depend 2015;153:43-9.

38. Farrell M, Marsden J. Acute risk of drug-related death among newly released prisoners in England and Wales. Addiction 2008;103:251-5.

39. Massoglia M, Remster B, King RD. Stigma or separation? Understanding the incarceration-divorce relationship. Social Forces 2011:90:133-55.

40. Thibodeau L, Jorenby DE, Seal DW, et al. Prerelease intent predicts smoking behavior postrelease following a prison smoking ban. Nicotine Tob Res 2010;12:152-8.

41. Puljevic C, Segan CJ. Systematic Review of Factors Influencing Smoking Following Release from Smoke-free Prisons. Nicotine Tob Res 2018

42. Cancer Council Victoria. Pilot Evaluation: Pre- and Post-Release Smoking Cessation Support for Victorians Transitioning from SmokeFree Prisons. Melbourne, Australia 2016.

43. Lindson-Hawley N, Thompson TP, Begh R. Motivational interviewing for smoking cessation. Cochrane Database Syst Rev 2015;3:1-76.

44. Wagner FA, Sheikhattari P, Buccheri J, et al. A Community-Based Participatory Research on Smoking Cessation Intervention for Urban Communities. J Health Care Poor Underserved 2016;27:35-50.

45. Chan A, Tetzlaff J, Altman D, et al. Research and reporting methods annals of internal medicine spirit 2013 statement: Defining standard protocol items for clinical trials. Ann Intern Med 2013;158:200-7.

46. West $R$, Raw M, McNeill A, et al. Health-care interventions to promote and assist tobacco cessation: a review of efficacy, effectiveness and affordability for use in national guideline development. Addiction 2015;110:1388-403. 
47. Kotz D, Brown J, West R. "Real-world" effectiveness of smoking cessation treatments: a population study. Addiction 2014;109:491-9.

48. Stead LF, Hartmann-Boyce J, Perera R, et al. Telephone counselling for smoking cessation. Cochrane Database Syst Rev 2013;100.

49. Borland R, Segan CJ, Livingston PM, et al. The effectiveness of callback counselling for smoking cessation: a randomized trial. Addiction 2001;96:881-9.

50. Segan CJ, Maddox S, Borland R. Homeless clients benefit from smoking cessation treatment delivered by a homeless persons' program. Nicotine Tob Res 2015;17:996-1001.

51. World Health Organization. Training for tobacco quit line counsellors: Telephone counselling. Geneva, Switzerland 2014.

52. Stead L, Perera R, Bullen C, et al. Nicotine replacement therapy for smoking cessation. Cochrane Database Syst Rev 2012;11:4-6.

53. Hansson A, Hajek P, Perfekt R, et al. Effects of nicotine mouth spray on urges to smoke, a randomised clinical trial. BMJ Open 2012;2:e001618-6.

54. Tønnesen P, Lauri H, Perfekt R, et al. Efficacy of a nicotine mouth spray in smoking cessation: a randomised, double-blind trial. Eur Respir J 2012;40:548-54.

55. Australian Government Department of Health. The Pharmaceutical Benefits Scheme. Canberra, Australia 2018 http://www.pbs.gov.au/ $\mathrm{pbs} / \mathrm{home}$.

56. Bonevski B, Bryant J, Paul C. Encouraging smoking cessation among disadvantaged groups: a qualitative study of the financial aspects of cessation. Drug Alcohol Rev 2011;30:411-8.

57. PBS. Pharmaceutical Benefits Scheme A-Z Medicine Listing, 2017. Published. http://www.pbs.gov.au/browse/medicine-listing.

58. Royal Australian College of General Practitioners. Supporting Smoking Cessation: A Guide for Health Professionals. Melbourne, Australia 2011.

59. Australian Government Department of Health. Pharmaceutical Benefits Scheme Medicine Listing: Nicotine. http://www.pbs.gov.au/ medicine/item/4571N (Accessed 12 Apr 2018).

60. Puljević C, de Andrade D, Carroll M, et al. Use of prescribed smoking cessation pharmacotherapy following release from prison: a prospective data linkage study. Tob Control 2018;27.

61. Kinner SA, Alati R, Longo M, et al. Low-intensity case management increases contact with primary care in recently released prisoners: a single-blinded, multisite, randomised controlled trial. J Epidemiol Community Health 2016;70:683-8.

62. Young JT, Arnold-Reed D, Preen D, et al. Early primary care physician contact and health service utilisation in a large sample of recently released ex-prisoners in Australia: prospective cohort study. BMJ Open 2015;5:e008021.

63. Borland R, Segan CJ. The potential of quitlines to increase smoking cessation. Drug Alcohol Rev 2006;25:73-8.

64. Smith AL, Chapman S, Dunlop SM. What do we know about unassisted smoking cessation in Australia? A systematic review, 2005-2012. Tob Control 2015;24:18-27.

65. Bradley KA, DeBenedetti AF, Volk RJ, et al. AUDIT-C as a brief screen for alcohol misuse in primary care. Alcohol Clin Exp Res 2007;31:1208-17.

66. Humeniuk R, Henry-Edwards S, Ali R, et al. The Alcohol, Smoking and Substance Involvement Screening Test (ASSIST): Manual for Use in Primary Care. Geneva, Switzerland 2010.

67. Cappelleri JC, Bushmakin AG, Baker CL, et al. Revealing the multidimensional framework of the Minnesota nicotine withdrawal scale. Curr Med Res Opin 2005;21:749-60.
68. Kessler RC, Andrews G, Colpe LJ, et al. Short screening scales to monitor population prevalences and trends in non-specific psychological distress. Psychol Med 2002;32:959-76.

69. Sobell LC, Sobell MB. Measuring Alcohol Consumption. In: Litten RZ, Allen JP, eds. Totowa NJ: Humana Press, 1992.

70. Brown RA, Burgess ES, Sales SD, et al. Reliability and validity of a smoking timeline follow-back interview. Psychology of Addictive Behaviors 1998;12:101-12.

71. Courtney RJ, Naicker S, Shakeshaft A, et al. Smoking cessation among low-socioeconomic status and disadvantaged population groups: A systematic review of research output. Int J Environ Res Public Health 2015;12:6403-22.

72. Lawrence G, Dinh I, Taylor L. The centre for health record linkage: A new resource for health services research and evaluation. Health Inf Manag 2008;37:60-2.

73. Sibthorpe B, Kliewer E, Smith L. Record linkage in Australian epidemiological research: health benefits, privacy safeguards and future potential. Aust J Public Health 1995;19:250-6.

74. Kelman CW, Bass AJ, Holman CD. Research use of linked health data--a best practice protocol. Aust N Z J Public Health 2002;26:251-5.

75. AlHW. Data Integration Projects. 2017 http://www.aihw.gov.au/dataintegration/projects-2017/.

76. Sutherland G, Carroll M, Lennox N, et al. Prescribed contraceptives among woman after release from prison. Health Justice 2015;3:8

77. Borschmann R, Thomas E, Moran P, et al. Self-harm following release from prison: A prospective data linkage study. Aust $N Z J$ Psychiatry 2017;51:250-9.

78. Kinner SA, Forsyth S, Williams G. Systematic review of record linkage studies of mortality in ex-prisoners: why (good) methods matter. Addiction 2013;108:38-49.

79. Carroll M, Spittal MJ, Kemp-Casey AR, et al. High rates of general practice attendance by former prisoners: a prospective cohort study. Med J Aust 2017:207:75-80.

80. Kariminia A, Law MG, Butler TG, et al. Factors associated with mortality in a cohort of Australian prisoners. Eur J Epidemiol 2007;22:417-28

81. Kemp A, Preen DB, Saunders C, et al. Ascertaining invasive breast cancer cases; the validity of administrative and selfreported data sources in Australia. BMC Med Res Methodol 2013;13:17.

82. Hughes JR, Keely JP, Niaura RS, et al. Measures of abstinence in clinical trials: issues and recommendations. Nicotine Tob Res 2003;5:13-26.

83. Maclaren DJ, Conigrave KM, Robertson JA, et al. Using breath carbon monoxide to validate self-reported tobacco smoking in remote Australian Indigenous communities. Popul Health Metr 2010;8:2.

84. Puljević C, Coomber R, de Andrade D, et al. Barriers and facilitators of maintained smoking abstinence following release from smoke-free prisons: A qualitative enquiry. International Journal of Drug Policy 2019;68:9-17.

85. David MC, Alati R, Ware RS, et al. Attrition in a longitudinal study with hard-to-reach participants was reduced by ongoing contact. $J$ Clin Epidemiol 2013;66:575-81.

86. Mukaka M, White SA, Terlouw DJ, et al. Is using multiple imputation better than complete case analysis for estimating a prevalence (risk) difference in randomized controlled trials when binary outcome observations are missing? Trials 2016;17:341. 